\title{
Covariant higher order perturbations of branes in curved spacetime
}

\author{
Riccardo Capovilla๑, ${ }^{*}$ Giovany Cruz $\odot,{ }^{\dagger}$ and Edgar Yair López® \\ Departamento de Física, Cinvestav-IPN, Avenida Instituto Politécnico Nacional 2508, \\ colonia San Pedro Zacatenco, 07360 Gustavo A. Madero, Ciudad de México, México
}

(Received 20 October 2021; accepted 21 December 2021; published 11 January 2022)

\begin{abstract}
The treatment of higher order perturbations of branes is considered using a covariant variational approach. This covariant variational approach brings to the forefront the geometric structure of the underlying perturbation theory, as opposed to a more commonly used "direct approach," that ignores the variational origin. In addition, it offers a clear calculational advantage with respect to so-called "gauge fixed" treatments that distinguish tangential and normal modes, as it emphasizes the symmetries of the geometric models that describe the brane dynamics. We restrict our attention to a brane action that depends at most on first derivatives of the embedding functions of the world volume spanned by the brane in its evolution. We consider first and second variations of the action that describes the brane dynamics. The first variation produces the equations of motion, as is well known. In the second variation we derive the Jacobi equations for these kind of models, and we emphasize the role of the Hessian matrix. This is extended to third order in variations, first in a flat and then in a curved spacetime background. Further, we specialize to the relevant case of the Dirac-Nambu-Goto action that describes extremal branes. The proper setting of a covariant variational approach allows to go in principle to geometric models that depend on higher derivatives of the embedding functions, and higher order perturbations, with the due complications involved, but with a solid framework in place.
\end{abstract}

DOI: 10.1103/PhysRevD.105.025011

\section{INTRODUCTION}

Brane mechanics refers to the study of the dynamics of branes embedded in a higher dimensional space, usually called ambient space, background space, or target space. This is a generalization of the notion of a relativistic particle, a 0-brane. Then we can have strings or 1-branes. The term branes is leaving the dimension arbitrary. Branes are highly relevant in the description of different physical scenarios where the relevant physical degrees of freedom are confined to an appropriate subregion of interest. Branes are described by a local action that is a functional of the geometry of the world volume spanned by the brane in its evolution. The guiding principles in the construction of the action are reparametrization invariance and background diffeomorphism invariance. This turns the action into a geometric model. The term "brane mechanics" was introduced by Carter [1,2], where it is emphasized the range of

\footnotetext{
capo@ fis.cinvestav.mx †cruz@fis.cinvestav.mx *elopez@fis.cinvestav.mx
}

Published by the American Physical Society under the terms of the Creative Commons Attribution 4.0 International license. Further distribution of this work must maintain attribution to the author(s) and the published article's title, journal citation, and DOI. Funded by SCOAP. applications of the subject. Since then, the range has been widely extended in important ways.

At the classical level, even the simplest geometric models produce equations of motion that are highly nonlinear. An important special case are strings propagating in a flat background, where the equations of motion turn out to be linear, in an appropriate gauge [3]. Besides this special case, in general one confronts a serious challenge in the analysis of the possible solutions of the equations of motion. In addition, the nonlinearity can be made even worse if one considers a nontrivial background spacetime, with nonvanishing curvature, see, e.g., [4] for the case of a string, where the background curvature plays the role of an external force. In face of these difficulties, one has to resort to perturbation theory. The first step is to encounter a relevant exact solution to perturb about. To attain this, symmetries are invoqued and exploited, to make the problem tractable. The next obvious step is a direct linearization of the equations of motion that produce a set of equations for field deviations of fields that (on-shell) reduce to coupled quadratic oscillators, and so on. This "direct approach" is the one more commonly used in the literature [5]. There is also a "covariant direct approach" that exploits a perturbation taken as a directional covariant derivative along a deviation vector, or deformation, and that is especially advantageous in a curved background, see, e.g., Ref. [6]. 
The main subject of this paper is to offer a complete covariant variational approach that does not use any gaugefixing at any stage, where by gauge-fixing it is meant a split of brane perturbations in their normal and tangential modes with respect to the world volume. This approach sets brane mechanics as a covariant classical field theory, trying to avoid any idiosyncratic notation, and using standard familiar language, that hopefully will be useful to the reader. The covariance is with respect to both background diffeomorphisms and world volume reparametrization invariance. The main tool that we exploit is a covariant variational derivative, inspired by the pioneering work of Bażański [7], and its higher order extensions. From this point of view, conservation laws become easily accessible via Noether's theorem, as well as how they behave under perturbations [8]. In general, one has at its disposal the whole weaponry of the calculus of variations, properly generalized to a field theory. The emphasis on covariance is not only desirable, but needed because gauge invariance is a subtle point in perturbation theory. As one goes to higher orders in perturbation theory, it becomes more and more difficult to disentangle physical modes from gauge unphysical modes. As emphasized especially by Carter, explicit covariance allows one to sidestep many artificial complications. In any case, once covariant expressions are obtained, with reasonable minimal pain, one has always the option to gauge-fix to one heart's desire. In the classical case this is especially important to get to a seed solution of physical interest. Perturbations then would need to respect the gauge fixing conditions. When considering possible quantum corrections, it is mandatory in order to arrive to a proper definition of physical states.

The action for the brane is defined as an integral over the world volume of a Lagrangian density of weight one, to ensure reparametrization invariance. Its first order variation produces the Euler-Lagrange derivative and a Noether current. The latter is instrumental in the derivation of the canonical linear momentum, that will play a central role in our approach. The vanishing of the first variation, under suitable boundary conditions, gives the equations of motion for the system. The second order variation of the action has been considered by many authors in a "direct approach." This corresponds to a linearization of the equations of motion $[4,9,10]$. An approach related to ours has been offered in the important work by Battye and Carter [11]. The motivation to go to second order is manifold, see e.g., Ref. [12] for hypersurfaces in arbitrary spacetimes. In brane mechanics, the second order variation is used to study stability of solutions, see, e.g., Ref. [13] for a covariant study of perturbations in Lovelock type brane gravity. In addition, like the geodesic deviation equation for a relativistic particle, the vanishing of the second variation, onshell, produces Jacobi equations that describe the relative motion of neighboring branes connected by a deviation vector. In a curved background, they take the form of a kinetic term that involves the Hessian for the model, with the background Riemann curvature playing the role of an external force. The second variation also gives the index of the geometric model. The index is of paramount importance in various geometrical contexts, including geometry in the large, see e.g., Ref. [14] for minimal surfaces. In addition, in the study of quantum or thermal fluctuations the second variation appears as the "shape operator" that in a path integral determines both one-loop contributions and the semiclassical regime.

In many instances, second order variations is not enough, as degeneracies may emerge, and a third variation of the action is needed. In any case, it is useful to establish a pattern in perturbation theory that potentially can be extended to higher orders. This should be of interest especially in relativistic astrophysical applications, where observational data are becoming more and more precise, and a covariant perturbation theory is a needed theoretical tool. In this context, the works $[15,16]$ are a recent attempt to match upcoming cosmological data with perturbative theoretical models beyond general relativity. The third variation we derive exhibits an interesting structure, with a kinetic term that depends on the Jacobi operator, inherited from the second variation, acting on the second order perturbations, and a source term that depends on the known first order perturbations. This structure was known previously in classical field theory, see Ref. [7]. One previous study on second order perturbation of Dirac-Nambu-Goto (DNG) branes in a curved background by Kiosses and Nicolaidis needs to be mentioned in this context [17] (see also Ref. [18]). As far as we know, it was the first attempt to go to a description of second order perturbations in full generality. Their strategy is a direct approach splitting the perturbations in parts tangential and normal to the world volume. Although extremely convenient at first order, where tangential deformations can be associated to a mere reparametrization, and ignored safely, at higher order things become quite complicated, and the risk is to miss the forest for the trees. A covariant variational approach allows to understand the geometric structure of perturbation theory. In addition, we consider any codimension for the brane, although codimension one, for a hypersurface and codimension two are simpler to deal with. The presence of source terms that depend not only on the background Riemann curvature, but also on its covariant derivative is identified.

In recent years, extremal surfaces and their perturbations have emerged as actors in the context of AdS/CFT or gauge/gravity duality. In [19] it was conjectured that the entanglement entropy of a spatial subregion in a CFT can be calculated perturbatively using the area of a codimension-2 spacelike minimal surface in the dual gravity theory. A covariant version of this holographic entanglement entropy for arbitrary regions and "extremal" surfaces was given in [20]. Based on this, the authors of [21,22] 
argued that the Jacobi operator for two-dimensional surfaces in a particular coordinate system is uniquely fixed using only boundary data. Classically in the gravity side, holographic entanglement entropy is proportional to a surface area. However, probably this is not the whole story. One may include quantum fields adding to the entanglement, that in turn transform the extremal surfaces into "quantum extremal surfaces." They are defined as stationary points of Bekenstein's generalized entropy [23]. Branes have also been considered for the physics of the large. One imagines the observable universe as being a brane embedded in a higher dimensional place, with all of its fields and particles constrained to the brane, only gravity is free to "leak" into the bulk at high energies. These braneworld models offer possible phenomenological corrections to general relativity $[24,25]$.

There are other contexts, unrelated, where a brane geometrical description is useful and has been proved very successful both theoretically and in its experimental confirmation in the description of equilibrium configurations of lipid membranes in soft matter physics, see e.g., Ref. [26] and references therein. There is a common geometrical language, but with respect to more speculative relativistic settings, there is also the crucial advantage of experimental verification, to test theoretical endeavor. A geometric approach to fluid lipid membranes in space, using a covariant variational approach is proposed in $[27,28]$. Hydrodynamics on curved surfaces can be studied with a geometric description of submanifolds [29]. In this context, the theoretical work by Armas and collaborators is worth mentioning, e.g., in [30], a thorough study of brane and edge dynamics is given.

This paper is organized as follows. Section II gives a brief overview of the embedding geometry and sets our notation. Section III establishes a general covariant variational approach for relativistic brane models that depend at most on first order derivatives on the embedding functions. We construct systematically the first, second and third order variations of the action, by considering covariant variations with respect to a one parameter family of embeddings. For the sake of clarity, the third order variation is performed first in a flat background, then we add the complication of a curved background. Section IV applies the general formalism to the case of the DNG action. Finally, in Sec. V we offer a brief discussion.

\section{EMBEDDING GEOMETRY}

In this section, we describe briefly the geometry of the embedding of a time-like submanifold, or world volume, that depicts the evolution of a relativistic object, or brane, in a fixed background spacetime.

The semi-Riemannian background spacetime is $\left\{M, g_{\mu \nu}\right\}$ of dimension $N+1$ with local coordinates $x^{\mu}$ $(\mu, \nu, \ldots=0,1, \ldots, N)$, and endowed with a metric $g_{\mu \nu}$ of signature with mostly plus signs. Spacetime indices are lowered and raised with the spacetime metric $g_{\mu \nu}$ and its inverse $g^{\mu \nu}$. The background spacetime covariant derivative $\nabla_{\mu}$ is assumed to be torsionless and compatible with the background metric $g_{\mu \nu}, \nabla_{\rho} g_{\mu \nu}=0$, with Riemann curvature tensor defined as $\left[\nabla_{\mu}, \nabla_{\nu}\right] V^{\rho}=-R_{\mu \nu \sigma}{ }^{\rho} V^{\sigma}$, where we follow the conventions of [31].

The brane of dimension $d$ sweeps in its evolution a world volume $w$ of dimension $d+1$. The world volume $w$ can be described in a parametric form by the (timelike) local embedding functions $x^{\mu}=X^{\mu}\left(\xi^{a}\right)$, in $\left\{M, g_{\mu \nu}\right\}$, where $\xi^{a}$ are local coordinates for $w(a, b, \ldots=0,1, \ldots, d)$. The $d+1$ tangent vectors to the world volume $w$ are $X_{a}^{\mu}=\partial_{a} X^{\mu}=\partial X^{\mu} / \partial \xi^{a}$, and the induced metric, or first fundamental form, on $w$ is

$$
\gamma_{a b}=g_{\mu \nu} X_{a}^{\mu} X_{b}^{\nu}
$$

with determinant $\gamma$. The timelike character of the world volume is assured by requiring $\gamma<0$. Tangential indices are raised with the inverse induced metric $\gamma^{a b}$ and lowered with $\gamma_{a b}$. We denote with $\bar{\nabla}_{a}$ the world volume torsionless covariant derivative compatible with the induced metric $\gamma_{a b}, \bar{\nabla}_{a} \gamma_{b c}=0$, with Riemann curvature $\mathcal{R}_{a b c}{ }^{d}$.

The extrinsic geometry of the world volume can be introduced via the spacelike normal vectors $n^{\mu}{ }_{i}$ to the world volume $(i, j \ldots=1,2, \ldots, N-d)$ that are defined up to a sign and a rotation by the orthogonality condition $g_{\mu \nu} X_{a}^{\mu} n^{\nu}{ }_{i}=0$ and normalized with $g_{\mu \nu} n_{i}{ }_{i} n^{\nu}{ }_{j}=\delta_{i j}$, where $\delta_{i j}$ is the Kronecker delta. We leave the codimension arbitrary. Of course, there are noticeable simplifications for a hypersurface, $N-d=1$. We also note the completeness relationship

$$
g^{\mu \nu}=h^{\mu \nu}+n^{\mu \nu}=\gamma^{a b} X_{a}^{\mu} X_{b}^{\nu}+n^{\mu}{ }_{i} n^{\nu i},
$$

that defines the tangential and normal projectors $h^{\mu \nu}$ and $n^{\mu \nu}$, respectively.

The generalization of the classical Gauss-Weingarten equations for submanifolds to a curved background is given by

$$
\begin{gathered}
\nabla_{a} X_{b}^{\mu}=\gamma_{a b}{ }^{c} X_{c}^{\mu}-K_{a b}{ }^{i} n_{i}^{\mu}, \\
\tilde{\nabla}_{a} n^{\mu}{ }_{i}=K_{a c i} \gamma^{c b} X_{b}^{\mu} .
\end{gathered}
$$

Here $\gamma_{a b}{ }^{c}$ is the Christoffel symbol associated with the world volume covariant derivative $\bar{\nabla}_{a}$, and $\nabla_{a}=X_{a}^{\nu} \nabla_{\nu}$ is the projection of the background spacetime covariant derivative onto the world volume. The extrinsic curvature tensor, or second fundamental form, is

$$
K_{a b}{ }^{i}=-g_{\mu \nu} n^{\mu i} \nabla_{a} X_{b}^{\nu}=g_{\mu \nu} X_{b}^{\nu} \nabla_{a} n^{\mu i},
$$

and $K^{i}=\gamma^{a b} K_{a b}{ }^{i}$ denotes the mean extrinsic curvature. The twiddle on the covariant derivative of the normal vector 
in Eq. (4) denotes the $O(N-d)$ rotation covariant derivative $\tilde{\nabla}_{a} n^{\mu}{ }_{i}=\nabla_{a} n^{\mu}{ }_{i}-\omega_{a i}{ }^{j} n^{\mu}{ }_{j}$, where the connection $\omega_{a}{ }^{i j}=$ $-\omega_{a}{ }^{j i}$ is the twist potential, see e.g., Ref. [6]. In this brief introduction, we prefer a "kinematical" description, without getting into the more essential aspects of the embedding geometry, that involve a proper treatment of the relationship of the intrinsic and extrinsic geometry of the world volume, via the Gauss-Codazzi-Mainardi equations.

\section{GENERAL COVARIANT VARIATIONAL APPROACH}

We consider a geometric model that describes the dynamics of a relativistic brane that satisfies the requirements of reparametrization invariance and that is a scalar under background diffeomorphism transformations. In addition, we focus our attention on models that depend at most on first derivatives of the embedding functions

$$
S[X]=\int_{w} \mathcal{L}\left(X_{a}^{\mu}\right),
$$

where the Lagrangian density of weight one $\mathcal{L}\left(X_{a}^{\mu}\right)$ depends only on the intrinsic geometry of the world volume $w$. We have absorbed the differential $d^{d+1} \xi$ in the integral sign, henceforth. For the sake of simplicity we assume that the brane has no boundary, and with appropriate boundary conditions $\partial w=0$. The simplest example that will be the focus of our attention in the next section is the DNG model, proportional to the volume of $w$, with $\mu$ a constant tension,

$$
S_{D N G}[X]=-\mu \int_{w} \sqrt{-\gamma}
$$

In the following, we consider the first, second, and third variations of the general intrinsic geometric model (6). This general approach allows to establish a covariant perturbation theory that can be extended to more complex physical systems, once a proper framework for geometric models is introduced.

\section{A. First variation}

We consider an infinitesimal variation of the field variables, the embedding functions,

$$
X^{\mu} \rightarrow X^{\mu}+s \delta X^{\mu} .
$$

The standard field variation in the calculus of variations involves considering a one-parameter family of embedding functions $X^{\mu}\left(\xi^{a}, s\right)$, with $s$ an arbitrary parameter that labels the leaves of the family, and a coordinate field variation $\delta X^{\mu}=\left.\left(\partial X^{\mu}\left(\xi^{a}, s\right) / \partial s\right)\right|_{s=0}$, see, e.g., Ref. [32,33]. This "coordinate Taylor approach" is then extended to higher orders, in a systematic perturbation treatment. Although appropriate and sufficient for systems with a finite number of degrees of freedom, as in familiar classical mechanics systems, in field theory it is convenient to upgrade the variation to take into account the underlying symmetries. Thus, for our purposes, we adopt instead a covariant directional variational derivative

$$
\delta_{X}=\delta X^{\mu} \nabla_{\mu}
$$

along the variation $\delta X^{\mu}$. For a treatment of the covariant variational derivative see, e.g., [6,34], but we have not been able to identify other more complete studies on this particular subject in the available literature. The manifest convenience is that $\delta_{X} g_{\mu \nu}=0$, since the background covariant derivative is assumed to be metric compatible. Moreover, we assume from the outset that the variation is conserved along the world volume. Geometrically, this translates into the statement that the Lie derivative of the variation vector along the tangent vectors vanishes,

$$
\left[\delta_{X}, \nabla_{a}\right] X^{\mu}=\delta_{X} X_{a}^{\nu}-\nabla_{a} \delta X^{\nu}=0
$$

or, more prosaically, that variation and world volume partial derivative commute. This can be understood from the fact that we are considering a one-parameter family of embedding functions, labeled by $s$, and infinitesimally partial derivatives of the embedding functions commute, as required to have a foliation [32]. It should also be emphasized that we resist the temptation to split the variation $\delta X^{\mu}$ into its normal and tangential parts. Although this is extremely convenient in the first variation, since the tangential variation can be associated to a mere reparametrization, and in the absence of boundaries it can be safely neglected as pure gauge, at higher orders this early gauge fixing meddles the general structure of the perturbation theory. The usefulness of a spacetime covariant treatment in brane mechanics has been championed especially by Carter, see, e.g., Ref. [2] and references therein.

Turning to the first variation of the geometric model (6), we have simply

$$
\delta_{X} S[X]=\int_{w} \delta_{X} \mathcal{L}\left(X_{a}^{\mu}\right),
$$

where we use (10) to obtain

$$
\delta_{X} \mathcal{L}=\frac{\partial \mathcal{L}}{\partial X_{a}^{\mu}} \delta X_{a}^{\mu}=\frac{\partial \mathcal{L}}{\partial X_{a}^{\mu}} \nabla_{a} \delta X^{\mu} .
$$

Integration by parts of Eq. (11) gives immediately

$$
\begin{aligned}
\delta_{X} S[X] & =\int_{w} \frac{\partial \mathcal{L}}{\partial X_{a}^{\mu}} \nabla_{a} \delta X^{\mu} \\
& =\int_{w} \mathcal{E}_{\mu}(\mathcal{L}) \delta X^{\mu}+\int_{w} \nabla_{a} \mathcal{Q}^{a},
\end{aligned}
$$

where we identify the Noether current density 


$$
\mathcal{Q}^{a}=\frac{\partial \mathcal{L}}{\partial X_{a}^{\mu}} \delta X^{\mu}=\mathcal{P}_{\mu}{ }^{a} \delta X^{\mu},
$$

and the canonical linear momentum, a spacetime one-form and a world volume vector density,

$$
\mathcal{P}_{\mu}{ }^{a}=\frac{\partial \mathcal{L}}{\partial X_{a}^{\mu}} .
$$

The Euler-Lagrange derivative is

$$
\mathcal{E}_{\mu}(\mathcal{L})=-\nabla_{a}\left(\frac{\partial \mathcal{L}}{\partial X_{a}^{\mu}}\right)=-\nabla_{a} \mathcal{P}_{\mu}{ }^{a}
$$

As a consequence of reparametrization invariance, it can be expressed as a conservation law. The vanishing of the first variation (13), for arbitrary variations $\delta X^{\mu}$, produces the equations of motion

$$
\mathcal{E}_{\mu}(\mathcal{L})=0
$$

For intrinsic geometric models these equations of motion are of second order. This feature is shared by Lovelock branes, see, e.g., Ref. [35]. For models that depend on the extrinsic geometry, for example on the squared extrinsic curvature, the background spacetime curvature appears already in the equations of motion [2,6]. Higher derivative geometric models can be studied in a covariant framework, but the reader should be warned that the treatment becomes quite complicated from the outset, even in a flat background spacetime. Yet, all the tools are available, and it can be carried through, see, e.g., Ref. [13].

\section{B. Second variation}

Once one has calculated the first variation, the second variation is the natural next step and it plays a relevant role in many physical contexts. For example, when one is studying structure formation in our Universe, or anisotropies in the cosmic microwave background. In brane scenarios, the second variation is also important to study the stability of branes and know the behavior of their intrinsic geometry when a deformation is done. Besides, in a possible quantization via path integral of this kind of geometrical models, the second variation will be important to calculate the quantum correction to one loop.

For the second variation of the geometric model (6), we choose to exploit the expression (13) for the first variation. For the sake of simplicity, we assume that the world volume has no boundary, $\partial w=0$, or that appropriate boundary conditions have been chosen, and we neglect the Noether current contribution for the moment.

In the standard calculus of variations, one considers a one parameter family of embeddings $X^{\mu}\left(\xi^{a} ; s\right)$ and a coordinate variation

$$
\delta^{2} X^{\mu}=\left(\frac{\partial^{2} X^{\mu}\left(\xi^{a} ; s\right)}{\partial s^{2}}\right)_{s=0} .
$$

As in the case of the first variation, we adopt a second order covariant variational derivative, with appropriate labels, $\delta_{X}^{2}=\delta X^{\mu} \nabla_{\mu}\left(\delta X^{\nu} \nabla_{\nu}\right)$. Then, the second variation can be written as

$$
\delta_{X}^{2} S[X]=\int_{w}\left[\left(\delta_{X} \mathcal{E}_{\mu}(\mathcal{L})\right) \delta X^{\mu}+\mathcal{E}_{\mu}(\mathcal{L}) \delta^{2} X^{\mu}\right] .
$$

Assuming that the equations of motion (17) are satisfied the second term vanishes, and this expression reduces to

$$
\left.\delta_{X}^{2} S[X]\right|_{[0]}=\int_{w}\left[\left.\delta_{X} \mathcal{E}_{\mu}(\mathcal{L})\right|_{[0]}\right] \delta X^{\mu},
$$

where the subscript [0] is a reminder that we are on-shell. The evaluation of the second variation amounts to a linearization of the equations of motion about a solution, and this is the path chosen by various authors, with a direct evaluation of $\left.\delta_{X} \mathcal{E}_{\mu}(\mathcal{L})\right|_{[0]}[4,9,10]$. In this note, we explore a different path, taking full advantage of the conservation law structure of the equations of motion. It should be emphasized that, in contrast to Eq. (10), when applying a variation to a world volume tensorial quantity it no longer commutes with a background covariant derivative. This is a consequence of the fact that the variation operator is a directional covariant derivative. Indeed, this is where the background spacetime curvature comes into play, as shown explicitly in the following. We have

$$
\begin{aligned}
\left.\delta_{X} \mathcal{E}_{\mu}(\mathcal{L})\right|_{[0]} & =-\delta_{X} \nabla_{a} \mathcal{P}_{\mu}{ }^{a} \\
& =-\left[\delta_{X}, \nabla_{a}\right] \mathcal{P}_{\mu}{ }^{a}-\nabla_{a} \delta_{X} \mathcal{P}_{\mu}{ }^{a} \\
& =-R_{\alpha \beta \mu}{ }^{\rho} \delta X^{\alpha} X_{a}^{\beta} \mathcal{P}_{\rho}{ }^{a}-\nabla_{a} \delta_{X} \mathcal{P}_{\mu}{ }^{a},
\end{aligned}
$$

where we have used the Bianchi identity in the first term, that acts on a spacetime one-form $\omega_{\rho}$ as $\left[\nabla_{\mu}, \nabla_{\nu}\right] \omega_{\rho}=$ $R_{\mu \nu \rho}{ }^{\sigma} \omega_{\sigma}$. Therefore the second variation (20) takes the form

$\left.\delta_{X}^{2} S[X]\right|_{[0]}=-\int_{w}\left[\nabla_{a} \delta_{X} \mathcal{P}_{\mu}{ }^{a}+R_{\alpha \beta \mu}{ }^{\rho} \delta X^{\alpha} X_{a}^{\beta} \mathcal{P}_{\rho}{ }^{a}\right] \delta X^{\mu}$.

Using the definition of the canonical momentum (15), the first term can be written as its variation, or linearization,

$$
\begin{aligned}
\delta_{X} \mathcal{P}_{\mu}{ }^{a} & =\frac{\delta^{2} \mathcal{L}}{\partial X_{b}^{\nu} \partial X_{a}^{\mu}}\left(\delta_{X} X_{b}^{\nu}\right) \\
& =\mathcal{H}_{\nu \mu}^{b a}\left(\delta_{X} X_{b}^{\nu}\right) \\
& =\mathcal{H}_{\nu \mu}^{b a}\left(\nabla_{b} \delta X^{\nu}\right),
\end{aligned}
$$

where we have defined the Hessian matrix 


$$
\mathcal{H}_{\nu \mu}^{b a}=\frac{\delta^{2} \mathcal{L}}{\partial X_{b}^{\nu} \partial X_{a}^{\mu}},
$$

and in the last equality we have used the fact that variation and partial derivative commute, see Eq. (10). Note that the Hessian is degenerate, it admits null eigenvectors, because of the gauge freedom associated with reparametrization invariance. The Hessian is symmetric in mixed pairs of indices

$$
\mathcal{H}_{\nu \mu}^{b a}=\mathcal{H}_{\mu \nu}^{a b} .
$$

Inserting Eq. (23) in the second variation (22), one obtains the general expression

$$
\begin{aligned}
\left.\delta_{X}^{2} S[X]\right|_{[0]}= & -\int_{w}\left\{\nabla_{a}\left[\mathcal{H}_{\nu \mu}^{b a}\left(\nabla_{b} \delta X^{\nu}\right)\right]\right. \\
& \left.+R_{\alpha \beta \mu}{ }^{\rho} \delta X^{\alpha} X_{a}^{\beta} \mathcal{P}_{\rho}{ }^{a}\right\} \delta X^{\mu} .
\end{aligned}
$$

All that is left to do is the calculation of the Hessian matrix, for a given geometric model defined by a specific $\mathcal{L}$.

At this point, $\delta X^{\mu}$ is arbitrary, and represents a linearized perturbation around any on-shell embedding. However, if we are interested in perturbations from one on-shell configuration to another, we need to choose $\delta X^{\mu}$ so that the second perturbation vanishes. From Eq. (26) we can immediately read the necessary nontrivial set of equations for the vector $\delta X^{\mu}$. These set of equations are known as Jacobi equations. We set the vanishing of the second variation in the form

$$
\left.\delta_{X}^{2} S[X]\right|_{[0]}=0,
$$

for the variation $\delta X^{\mu}$, that we can identify as a world volume "deviation vector," $\delta X^{\mu}=\eta^{\mu}$, interpreted as a linear approximation vector connecting neighboring branes in their evolution, just like in the familiar case of the geodesic deviation equation (see, e.g., [7,31,36]). Expression (27) gives the Jacobi equations for the deviation vector $\eta^{\mu}$ in the form

$$
-\nabla_{a}\left[\mathcal{H}_{\nu \mu}^{b a} \nabla_{b} \eta^{\nu}\right]-R_{\nu \beta \mu}{ }^{\rho} X_{a}^{\beta} \mathcal{P}_{\rho}{ }^{a} \eta^{\nu}=0,
$$

the first "kinetic" term is of second order, as expected, since the equations of motion are of second order. It displays the central role of the Hessian as a sort of "mass-matrix" in the language of classical mechanics. The second term, involving the background spacetime, can be seen as an external force. This is consistent with our intuition based on the geodesic deviation equation in general relativity [31]. It is worth noticing that in the case of a flat background, the Jacobi equations also take the form of a conservation law, as a divergence-free equation for the linearized canonical momentum. For an alternative, and more economic, avenue to the brane Jacobi equations in a curved background spacetime, using a covariant simultaneous variational principle, see Ref. [37].
Going back to Eq. (26), another thing we can do is an integration by parts of the first term, which produces, neglecting a boundary term,

$$
\begin{aligned}
\left.\delta_{X}^{2} S[X]\right|_{[0]}= & \int_{w}\left\{\mathcal{H}_{\nu \mu}^{b a}\left(\nabla_{b} \delta X^{\nu}\right)\left(\nabla_{a} \delta X^{\mu}\right)\right. \\
& \left.-R_{\alpha \beta \mu}{ }^{\rho} \delta X^{\alpha} X_{a}^{\beta} \mathcal{P}_{\rho}{ }^{a} \delta X^{\mu}\right\} \\
= & \int_{w} \mathcal{I}(\delta X, \delta X) .
\end{aligned}
$$

This expression identifies the quadratic form, or index, $\mathcal{I}(\delta X, \delta X)$, that is of fundamental importance in establishing stability of the solutions of the equations of motion, see, e.g., Ref. [32] in the calculus of variations, and [14] for minimal surfaces in Euclidean three-dimensional ambient space. The index may be evaluated at two different deviation vectors, $\mathcal{I}\left(\delta X_{(1)}, \delta X_{(2)}\right)$, corresponding with two different deviation directions. Usually the variations are identified, $\delta X_{(2)}=\delta X_{(1)}=\eta$, and one of the main difficulties in a proper analysis of the quadratic form lies in finding an appropriate complete basis that satisfies the required boundary conditions, depending on the problem at hand, to allow for a proper understanding of the quadratic form $[32,38]$. This idea of using different deviation vectors, and explore how it affects the stability analysis, will be considered in future work. The expression (29) is, except for factors of one half, identical to the Jacobi accessory variational principle, that provides an action whose extremalization gives the Jacobi equation as the vanishing of its Euler-Lagrange derivative, see, e.g., Ref. [33].

\section{Third variation}

In order to establish the usefulness of a covariant variational approach to perturbation theory for the dynamics of branes, we extend our treatment to third order. In addition, a reason to recur to the third variation of the geometric model is a possible degeneracy of the contribution at second order between deformation modes. As expected, the complexity of the calculation increases noticeably, yet a covariant variational approach allows to appreciate the underlying mathematical variational structure, and makes it ready for use in concrete applications when second order perturbations are included. Moreover, the covariant approach clarifies the interplay between first order and second order perturbations. For a previous study of these issues, see Ref. [39].

We consider the expression (20) for the second variation of the action. An additional variation [corresponding to an additional derivative in Eq. (18)], gives

$$
\begin{aligned}
\left.\delta_{X}^{3} S[X]\right|_{[0]}= & \int_{w}\left\{\left[\left.\delta_{X}^{2} \mathcal{E}_{\mu}(\mathcal{L})\right|_{[0]}\right] \delta X^{\mu}\right. \\
& \left.+\left[\left.\delta_{X} \mathcal{E}_{\mu}(\mathcal{L})\right|_{[0]}\right] \delta^{2} X^{\mu}\right\}
\end{aligned}
$$


Assuming that both the equations of motion (17) and the Jacobi equations (28) are satisfied, then the second term vanishes, and the third variation (29) reduces to

$$
\left.\delta_{X}^{3} S[X]\right|_{[0,1]}=\int_{w}\left[\left.\delta_{X}^{2} \mathcal{E}_{\mu}(\mathcal{L})\right|_{[0,1]}\right] \delta X^{\mu},
$$

where now the subscript $[0,1]$ is a reminder that, besides the equations of motion, also the Jacobi equations are assumed to be fulfilled. It remains to unpack the second variation of the equations of motion.

For this purpose, it is convenient to first assume a flat background, with vanishing Riemann tensor, and then in a second step introduce the complication of a curved background.

\section{Flat background spacetime}

Within the special case of a Minkowski spacetime, background derivatives commute, and we exploit, just like for the second variation, the fact that the equations of motion can be written as a conservation law.

In terms of the linear momentum, the second variation is given by Eq. (22). Therefore, using Eq. (15), the third variation is given by

$$
\left.\delta_{X}^{3} S[X]\right|_{[0,1]}=\left.\int_{w}\left[\left(\nabla_{a} \delta^{2} \mathcal{P}_{\mu}{ }^{a}\right) \delta X^{\mu}\right]\right|_{[0,1]},
$$

since partial derivative and variation commute. To unpack the second variation of the linear momentum, we use Eq. (23) for the first variation of the linear momentum, and remark that the variation, when acting on the first variation of the shape functions $\delta X$ gives a second variation of the shape functions $\delta^{2} X$, and when acting on the Hessian matrices of the energy density gives a source term that depends quadratically on the first variations $\delta X$. It is important to remember that the assumption that the Jacobi equation is satisfied is equivalent to assume that the first order perturbations $\delta X$ are given. In this sense, we are talking of a source term. The second variation of the linear stress tensor, as given by the variation of Eq. (23), can then be written as the sum

$$
\delta_{X}^{2} \mathcal{P}_{\mu}{ }^{a}\left(\delta^{2} X, \delta X\right)=\delta_{X} \mathcal{P}_{\mu}{ }^{a}\left(\delta^{2} X\right)+\mathcal{S}_{\mu}{ }^{a}(\delta X) .
$$

The first term depends on the second variation $\delta^{2} X$, and it has the same structure of the first order variation. It is given by the Jacobi operator, with $\delta X \rightarrow \delta^{2} X$ in Eq. (23). This term does not require any additional work, since the Jacobi operator is known, see Eq. (28). The second term in Eq. (32) is a source term that depends on the first variation $\delta X$, due to the variation of the Hessian matrix,

$$
\mathcal{S}_{\mu}{ }^{a}(\delta X)=\left(\frac{\partial^{2} \delta \mathcal{L}}{\partial X_{a}^{\mu} \partial X_{c}^{\nu}}\right) \nabla_{c} \delta X^{\nu}
$$

This term does require additional work. Using Eq. (12) for $\delta \mathcal{L}$, it takes the formidable aspect

$$
\mathcal{S}_{\mu}{ }^{a}(\delta X)=\mathcal{T}_{\rho \nu \mu}^{c b a} \nabla_{c} \delta X^{\nu} \nabla_{b} \delta X^{\rho},
$$

where we have defined the tensor of third order derivatives analogous to the Hessian

$$
\mathcal{T}_{\rho \nu \mu}^{c b a}=\frac{\partial^{3} \mathcal{L}}{\partial X_{c}^{\rho} \partial X_{b}^{\nu} \partial X_{a}^{\mu}} .
$$

Returning to the third variation of the action (31), we find therefore that it can be written in the deceivingly simple form

$\left.\delta^{3} S[X]\right|_{[0,1]}=\left.\int_{w}\left\{\left[\mathcal{J}_{\mu}\left(\delta^{2} X\right)+\nabla_{a} \mathcal{S}_{\mu}{ }^{a}(\delta X)\right] \delta X^{\mu}\right\}\right|_{[0,1]}$,

where the second order perturbations appear in the Jacobi operator, $\mathcal{J}_{\mu}\left(\delta^{2} X\right)=\nabla_{a} \delta \mathcal{P}_{\mu}{ }^{a}\left(\delta^{2} X\right)$, and the first order perturbations in the source term $\nabla_{a} \mathcal{S}_{\mu}{ }^{a}(\delta X)$. What can be appreciated from this expression for the third variation is that the higher order Jacobi equation it implies has a very different structure than the second order Jacobi equation. In particular, it is not homogenous, for the presence of a source term. Also for the third variation one can derive an accessory variational principle and a parallel simultaneous variational principle.

\section{Curved background spacetime}

Let us now consider how the third variation (36) is modified when the brane evolves in a curved background, when partial derivatives and variations do not commute. We start with equation (26) for the second variation with a nonvanishing curvature term. An additional third variation yields

$$
\begin{aligned}
\left.\delta_{X}^{3} S[X]\right|_{[0,1]}= & -\int_{\omega}\left\{\nabla_{a}\left[\delta_{X}\left(\mathcal{H}_{\nu \mu}^{b a} \nabla_{b} \delta X^{\nu}\right)\right]\right. \\
& +R_{\alpha \beta \nu}{ }^{\rho} \delta X^{\alpha} X_{a}^{\beta} \delta_{X} \mathcal{P}_{\rho}{ }^{a} \\
& \left.\times \delta_{X}\left(R_{\alpha \beta \mu}{ }^{\rho} \delta X^{\alpha} X_{a}^{\beta} \mathcal{P}_{\rho}{ }^{a}\right)\right\} \delta X^{\mu},
\end{aligned}
$$

where we used the Bianchi identity yet again in the first line. The first term can be written in terms of the canonical momentum using Eq. (23)

$$
\begin{aligned}
\delta_{X}\left(\mathcal{H}_{\nu \mu}^{b a} \nabla_{b} \delta X^{\nu}\right)= & \delta_{X}^{2} \mathcal{P}_{\mu}{ }^{a} \\
= & \mathcal{T}_{\rho \nu \mu}^{c b a}\left(\nabla_{c} \delta X^{\rho}\right)\left(\nabla_{b} \delta X^{\nu}\right) \\
& +\mathcal{H}_{\nu \mu}^{b a} \delta_{X}\left(\nabla_{b} \delta X^{\nu}\right) \\
= & \mathcal{T}_{\rho \nu \mu}^{c b a}\left(\nabla_{c} \delta X^{\rho}\right)\left(\nabla_{b} \delta X^{\nu}\right) \\
& +\mathcal{H}_{\nu \mu}^{b a} R^{\nu}{ }_{\alpha \rho \beta} \delta X^{\alpha} \delta X^{\rho} X_{b}^{\beta} \\
& +\mathcal{H}_{\nu \mu}^{b a} \nabla_{b} \delta^{2} X^{\nu},
\end{aligned}
$$

where the tensor $\mathcal{T}$ is defined in Eq. (35). In the last step, we commuted the derivative and the variation producing a 
background Riemann tensor projection. Let us now use the Leibniz rule to unpack the third term in Eq. (37),

$$
\begin{aligned}
\delta_{X}\left(R_{\alpha \beta \mu}{ }^{\rho} \delta X^{\alpha} X_{a}^{\beta} \mathcal{P}_{\rho}{ }^{a}\right)= & \left(\nabla_{\sigma} R_{\alpha \beta \mu}{ }^{\rho}\right) \delta X^{\sigma} \delta X^{\alpha} X_{a}^{\beta} \mathcal{P}_{\rho}{ }^{a} \\
& +R_{\alpha \beta \mu}{ }^{\rho}\left(\delta^{2} X^{\alpha}\right) X_{a}^{\beta} \mathcal{P}_{\rho}{ }^{a} \\
& +R_{\alpha \beta \mu}{ }^{\rho} \delta X^{\alpha} \nabla_{a}\left(\delta X^{\beta}\right) \mathcal{P}_{\rho}{ }^{a} \\
& +R_{\alpha \beta \mu}{ }^{\rho} \delta X^{\alpha} X_{a}^{\beta}\left(\delta_{X} \mathcal{P}_{\rho}{ }^{a}\right),
\end{aligned}
$$

where we used Eq. (10) in the second term. Notice that we now have many curvature terms along with a derivative of the Riemann tensor which severely complicates the expression, compared to the case of flat background spacetime. Inserting Eqs. (39) and (38) into Eq. (37), we get

$$
\begin{aligned}
\left.\delta_{X}^{3} S[X]\right|_{[0,1]}= & -\int_{w}\left\{\nabla _ { a } \left[\mathcal{T}_{\rho \nu \mu}^{c b a}\left(\nabla_{c} \delta X^{\rho}\right)\left(\nabla_{b} \delta X^{\nu}\right)\right.\right. \\
& \left.+\mathcal{H}_{\nu \mu}^{b a}\left(\nabla_{b} \delta^{2} X^{\nu}+R^{\nu}{ }_{\alpha \rho \beta} \delta X^{\alpha} \delta X^{\rho} X_{b}^{\beta}\right)\right] \\
& +2 R_{\alpha \beta \mu}{ }^{\rho} X_{a}^{\beta} \delta X^{\alpha} \delta_{X} \mathcal{P}_{\rho}^{a} \\
& +\left(\nabla_{\sigma} R_{\alpha \beta \mu}{ }^{\rho}\right) \delta X^{\sigma} \delta X^{\alpha} X_{a}^{\beta} \mathcal{P}_{\rho}{ }^{a} \\
& +R_{\alpha \beta \mu}{ }^{\rho}\left(\delta^{2} X^{\alpha}\right) X_{a}^{\beta} \mathcal{P}_{\rho}{ }^{a} \\
& \left.+R_{\alpha \beta \mu}{ }^{\rho} \delta X^{\alpha}\left(\nabla_{a} \delta X^{\beta}\right) \mathcal{P}_{\rho}{ }^{a}\right\} \delta X^{\mu} .
\end{aligned}
$$

As we can see, the first two lines are again a conservation law, now for the second variation of the canonical momentum.
Additionally, we have a term proportional to both the curvature and the first variation of the momentum, and a term linear in the second order deviation vector. Notice that now we have quadratic terms and derivatives of the first order deviation vector. It is assumed however, that such vector is known.

The vanishing of the previous integral leads to the equation

$$
\begin{gathered}
\nabla_{a}\left[\delta_{X}^{2} \mathcal{P}_{\mu}{ }^{a}\right]+R_{\alpha \beta \mu}{ }^{\rho} \delta X^{\alpha} X_{a}^{\beta} \delta_{X} \mathcal{P}_{\rho}{ }^{a} \\
+\delta_{X}\left(R_{\alpha \beta \mu}{ }^{\rho} \delta X^{\alpha} X_{a}^{\beta} \mathcal{P}_{\rho}{ }^{a}\right)=0,
\end{gathered}
$$

where we conveniently wrote it in terms of the canonical momentum, both to avoid unnecessarily long expressions and to show, as promised, the underlying structure of the equations. Here, the embeddings are known from the equations of motion, and the variation vector $\delta X^{\nu}$ satisfies the Jacobi equation (28) with $\eta^{\nu}=\delta X^{\nu}$. Equation (41) now determines the second order variation vector $\delta^{2} X^{\nu}$.

Like in the previous section, the variation (40) can be organized as follows

$$
\left.\delta_{X}^{3} S[X]\right|_{[0,1]}=-\int_{\omega}\left[\mathcal{J}_{\mu}\left(\delta^{2} X\right)+\mathcal{F}_{\mu}(\delta X)\right] \delta X^{\mu},
$$

where $\mathcal{F}_{\mu}$ is the source term given by

$$
\begin{aligned}
\mathcal{F}_{\mu}(\delta X)= & \nabla_{a}\left[\mathcal{T}_{\rho \nu \mu}^{c b a}\left(\nabla_{c} \delta X^{\rho}\right)\left(\nabla_{b} \delta X^{\nu}\right)+\mathcal{H}_{\nu \mu}^{b a} R^{\nu}{ }_{\alpha \rho \beta} \delta X^{\alpha} \delta X^{\rho} X_{b}^{\beta}\right]+\left(\nabla_{\sigma} R_{\alpha \beta \mu}{ }^{\rho}\right) \delta X^{\sigma} \delta X^{\alpha} X_{a}^{\beta} \mathcal{P}_{\rho}{ }^{a} \\
& +R_{\alpha \beta \mu}{ }^{\rho}\left[2 \delta X^{\alpha} X_{a}^{\beta} \mathcal{H}_{\nu \rho}^{b a} \nabla_{b} \delta X^{\nu}+\delta X^{\alpha} \nabla_{a} \delta X^{\beta} \mathcal{P}_{\rho}{ }^{a}\right] .
\end{aligned}
$$

Then, we can see that Eq. (41) is nothing but a Jacobi equation with source $\mathcal{F}_{\mu}$ for the second variation field $\delta^{2} X^{\mu}$, just like in Eq. (36). However, here the source term is modified by the curvature of the spacetime background.

The expression (41) has already been found in [17], but there only normal perturbation modes were considered, and the perturbations were done directly in the equations of motion. Here a fully covariant expression is given and seen from a variational perspective. This expression is a second order approximation to the deviation of neighboring onshell branes in a fixed arbitrary background. Going to second order perturbations is not only a natural next step after first order. There are other reasons to recur to second order perturbations such as overcoming degeneracies that appear at first order. Some physical quantities have a second order leading contribution. For a more extensive list, see, e.g., Ref. [12].

\section{DIRAC-NAMBU-GOTO BRANE}

The DNG action is a first approximation in this approach to constructing geometric models to describe physical systems, where we put to use the general covariant variational formulation we have described above. Originally, it was proposed by Dirac in a geometric relativistic model for an "extensible electron" [40]. Later, Nambu and independently Goto applied the same idea to a relativistic string to model gluons in QCD [41,42]. The rest is a long history.

From the viewpoint of brane mechanics, the DNG action is an obvious starting point. This action is proportional to the world volume of the brane and is invariant under diffeomorphisms. The DNG action is given by

$$
S_{D N G}[X]=\int_{w} \mathcal{L}_{D N G}=-\mu \int_{w} \sqrt{-\gamma},
$$

where $\mu$ is the membrane tension. Using the framework previously developed, for the first variation we only need the canonical momentum. The canonical momentum is

$$
\mathcal{P}_{\mu}{ }^{a}=\frac{\partial \mathcal{L}_{D N G}}{\partial X_{a}^{\mu}}=-\mu \sqrt{-\gamma} g_{\mu \nu} \gamma^{a b} X_{b}^{\nu} .
$$

Note that it is tangential to the world volume, as is the case for any intrinsic geometric model and isotropic. 
The equations of motion that are given by the vanishing of the first variation are

$$
\mathcal{E}_{\mu}\left(\mathcal{L}_{D N G}\right)=-\nabla_{a} \mathcal{P}_{\mu}{ }^{a}=-\mu \nabla_{a}\left(\sqrt{-\gamma} g_{\mu \nu} \gamma^{a b} X_{b}^{\nu}\right)=0
$$

Since the action is invariant under reparametrizations, the tangential variations do not influence the dynamics because these can be identified as a divergence. Thus when one projects the equations of motion onto the basis of the background spacetime, only the normal part survives, the latter is given by

$$
n^{\mu}{ }_{i} \nabla_{a} \mathcal{P}_{\mu}{ }^{a}=\mu \sqrt{-\gamma} K_{i}=0,
$$

i.e., the vanishing of the world volume mean extrinsic curvature $K_{i}=\gamma^{a b} K_{a b i}$. This is the relativistic version of the equilibrium condition for a minimal surface with arbitrary codimension.

For the second variation, we need only to calculate the Hessian,

$$
\mathcal{H}_{\nu \mu}^{b a}=\frac{\delta^{2} \mathcal{L}_{D N G}}{\partial X_{b}^{\nu} \partial X_{a}^{\mu}}=-\mu \sqrt{-\gamma}\left(\gamma^{a b} n_{\mu \nu}+X_{\mu \nu}^{a b}\right),
$$

where we have used the completeness relationship (2) that defines the normal projector $n^{\mu \nu}$. The tangential bivector that appears in Eq. (48) is

$$
X_{a b}^{\mu \nu}=2 X_{a}^{[\mu} X_{b}^{\nu]}
$$

with indices raised with $\gamma^{a b}$ and lowered with $g_{\mu \nu}$. Substituting in the general expression for the second variation (26) we have

$$
\begin{aligned}
\left.\delta_{X}^{2} S[X]\right|_{[0]}= & \mu \int_{w}\left\{\nabla_{a}\left[\sqrt{-\gamma}\left(\gamma^{a b} n_{\mu \nu}+X_{\mu \nu}^{a b}\right)\left(\nabla_{b} \delta X^{\nu}\right)\right]\right. \\
& \left.+\sqrt{-\gamma} R_{\alpha \beta \mu \rho} \delta X^{\alpha} h^{\beta \rho}\right\} \delta X^{\mu}
\end{aligned}
$$

where we identified the tangential projector defined in the completeness relation (2), given by

$$
h^{\beta \rho}=\gamma^{b c} X_{b}^{\beta} X_{c}^{\rho}
$$

For this case the Jacobi equations (28) read

$$
\begin{gathered}
\mu\left\{\nabla_{a}\left[\sqrt{-\gamma}\left(\gamma^{a b} n_{\mu \nu}+X_{\mu \nu}^{a b}\right)\left(\nabla_{b} \delta X^{\nu}\right)\right]\right. \\
\left.+\sqrt{-\gamma} R_{\alpha \beta \mu \rho} \delta X^{\alpha} h^{\beta \rho}\right\}=0 .
\end{gathered}
$$

Notice that normal and tangential projections of the deviation vector arise naturally in the expression, showing the mixture of both contributions. These equations provide a generalization to branes of the well-known geodesic deviation equation for particles.

For the third variation we need to obtain the tensor $\mathcal{T}$, defined in Eq. (35). When the algebraic dust settles down, we obtain

$$
\begin{aligned}
\mathcal{T}_{\tau \nu \mu}^{c b a}= & \sqrt{-\gamma}\left\{n_{\nu \tau}\left(\gamma^{b c} X_{\mu}^{a}-2 \gamma^{a b} X_{\mu}^{c}\right)\right. \\
& \left.+2 n_{\mu \tau}\left(\gamma^{a c} X_{\nu}^{b}-\gamma^{a b} X_{\nu}^{c}-\gamma^{b c} X_{\nu}^{a}\right)+X_{\mu \nu \tau}^{a b c}\right\}
\end{aligned}
$$

where there is an abundance of raised and lowered indices, and now we have a tangential antisymmetric trivector

$$
X_{\mu \nu \tau}^{a b c}:=3 ! X_{\mu}^{[a} X_{\nu}^{b} X_{\tau}^{c]}
$$

Putting it all together, we use the expressions (45), (48), and (53) to insert them into Eq. (42), taking into account Eq. (43). Then, we can write the full third variation for the DNG action

$$
\begin{aligned}
\left.\delta_{X}^{3} S_{D N G}\right|_{[0,1]}= & \int_{\omega}\left\{\mathcal{J}_{\mu}\left(\delta^{2} X\right)+\mu \nabla_{a}\left[\sqrt{-\gamma}\left(n_{\nu \tau}\left(\gamma^{b c} X_{\mu}^{a}-2 \gamma^{a b} X_{\mu}^{c}\right)+2 n_{\mu \tau}\left(\gamma^{a c} X_{\nu}^{b}-\gamma^{a b} X_{\nu}^{c}-\gamma^{b c} X_{\nu}^{a}\right)+X_{\mu \nu \tau}^{a b c}\right)\right.\right. \\
& \left.\times\left(\nabla_{c} \delta X^{\tau}\right) \nabla_{b}\left(\delta X^{\nu}\right)+\sqrt{-\gamma}\left(\gamma^{a b} n_{\mu \nu}+X_{\mu \nu}^{a b}\right) R_{\alpha \rho \beta}^{\nu} \delta X^{\alpha} \delta X^{\rho} X_{b}^{\beta}\right]+\mu \sqrt{-\gamma}\left(\nabla_{\omega} R_{\alpha \beta \mu \rho}\right) \delta X^{\omega} \delta X^{\alpha} h^{\beta \rho} \\
& \left.+\mu \sqrt{-\gamma} R_{\alpha \beta \mu}{ }^{\rho}\left[2 \delta X^{\alpha} X_{a}^{\beta} \nabla_{b}\left(\delta X^{\nu}\right)\left(\gamma^{a b} n_{\rho \nu}+X_{\rho \nu}^{a b}\right)+\delta X^{\alpha} \nabla_{a}\left(\delta X^{\beta}\right) g_{\rho \nu} \gamma^{a c} X_{c}^{\nu}\right]\right\} \delta X^{\mu}
\end{aligned}
$$

Of course, the vanishing of the last integral gives us the second order perturbation to the equations of motion

$$
\begin{aligned}
\mathcal{J}_{\mu}\left(\delta^{2} X\right)= & -\mu\left\{\nabla _ { a } \left[\sqrt { - \gamma } \left[\left(n_{\nu \tau}\left(\gamma^{b c} X_{\mu}^{a}-2 \gamma^{a b} X_{\mu}^{c}\right)+2 n_{\mu \tau}\left(\gamma^{a c} X_{\nu}^{b}-\gamma^{a b} X_{\nu}^{c}-\gamma^{b c} X_{\nu}^{a}\right)+X_{\mu \nu \tau}^{a b c}\right)\left(\nabla_{c} \delta X^{\tau}\right) \nabla_{b}\left(\delta X^{\nu}\right)\right.\right.\right. \\
& \left.\left.+\left(\gamma^{a b} n_{\mu \nu}+X_{\mu \nu}^{a b}\right) R_{\alpha \rho \beta}^{\nu} \delta X^{\alpha} \delta X^{\rho} X_{b}^{\beta}\right]\right]+\sqrt{-\gamma}\left(\nabla_{\omega} R_{\alpha \beta \mu \rho}\right) \delta X^{\omega} \delta X^{\alpha} h^{\beta \rho} \\
& \left.+\sqrt{-\gamma} R_{\alpha \beta \mu}{ }^{\rho}\left[2\left(\gamma^{a b} n_{\rho \nu}+X_{\rho \nu}^{a b}\right) \delta X^{\alpha} X_{a}^{\beta} \nabla_{b} \delta X^{\nu}+g_{\rho \nu} \gamma^{a c} X_{c}^{\nu} \delta X^{\alpha} \nabla_{a} \delta X^{\beta}\right]\right\},
\end{aligned}
$$


Here $\mathcal{J}_{\mu}\left(\delta^{2} X\right)$ is given by the left side of Eq. (52), with $\delta X \rightarrow \delta^{2} X$. The right side of Eq. (56) is the source term of Jacobi's nonhomogeneous equation. Notice that the source term is influenced by the Riemann tensor of the background spacetime and its covariant derivative. One might think that the covariant derivative on the Riemann tensor should vanish since the background spacetime is fixed. However, when making variations, local changes in the embeddings must be taken into account, which justifies the appearance of this term.

\section{DISCUSSION}

In this paper we have developed a systematic covariant variational approach to perturbation theory for intrinsic brane models. Perturbations are realized as covariant derivatives along the flow of a deformation vector, with respect to a one parameter family of the world volume corresponding to the brane's evolution. These vectors came from coordinate variations of a two parameters family of embeddings $X^{\mu}\left(\xi^{a} ; s\right)$. This is the standard approach used in the calculus of variations, but it has to be modified appropriately for systems with an infinite number of degrees of freedom.

As a first step, we consider models that depend only on the intrinsic geometry, i.e., that contain only first derivatives of the embedding functions. This set-up allows for a generalization to higher derivatives, relevant in many contexts, with the obvious complications involved.

We introduce a general covariant variational approach that to first order reproduces well-known equations of motion. At second order, we obtain the Jacobi equations that describe the evolution of deviation vectors that depict the behavior of a one-parameter family of branes under the external force provided by the background projected Riemann curvature. In the kinetic term that appears in the Jacobi equations it is emphasized the central role of the Hessian matrix of the geometrical model. In the special case of a DNG geometric model, the kinetic term takes the form of a mass matrix that separates neatly in a normal and tangential contribution. It should be mentioned at this point that a fully covariant approach is needed especially when considering boundary contributions. This consideration is relevant when issues of boundary vs. bulk contributions are deemed to be relevant. The Jacobi equation itself can be obtained from an accessory variational principle, as its Euler-Lagrange equation.

In our search for a suitable geometric structure for higher order variations, we extend the approach to a third variation of the action, making contact with previous investigations. What emerges is that the structure of the third variation is qualitatively different. The kinetic term is related to the Jacobi operator that appears in the second variation, but there is also a source term. This indicates the existence of a generic structure in perturbation theory that needs to be explored in more detail.

The equations obtained are very general, and can be applied readily to any specific background and any geometric model of interest. Unlike previous related works, we have not separated the perturbation into tangential and normal modes. Although this means we have nonphysical degrees of freedom in our results, one can always substitute normal deformations to eliminate this, recovering the results of previous works. The variational approach allowed us to notice that all perturbations may be expressed as conservation laws plus curvature terms.

The Dirac-Nambu-Goto action was taken as an example. We found that beyond first order, the tangential and normal projections of the deformation vectors are naturally present, even though the vector itself remained covariant. Even in this simpler case, the second order perturbation equations can be very complicated and the contribution of the spacetime curvature is highly relevant. The framework developed here can be applied to any intrinsic model that depends at most on first order derivatives of the embedding functions, the case for higher order dependance may be developed in a similar way.

\section{ACKNOWLEDGMENTS}

G. C. and E. Y. L. thank CONACyT for their graduate fellowships. R. C. thanks Sistema Nacional de Investigadores for partial support.
[1] B. Carter, Perturbation dynamics for membranes and strings governed by the Dirac-Nambu-Goto action in curved space, Phys. Rev. D 48, 4835 (1993).

[2] B. Carter, Essentials of classical brane dynamics, Int. J. Theor. Phys. 40, 2099 (2001).

[3] B. Zwiebach, A First Course in String Theory 2nd ed. (Cambridge University Press, Cambridge, England, 2009).
[4] A. L. Larsen and V. P. Frolov, Propagation of perturbations along strings, Nucl. Phys. B414, 129 (1994).

[5] A. Vilenkin and E.P.S. Shellard, Cosmic Strings and Other Topological Defects (Cambridge University Press, Cambridge, England, 1994).

[6] R. Capovilla and J. Guven, Geometry of deformations of relativistic membranes, Phys. Rev. D 51, 6736 (1995). 
[7] S. L. Bażański, Relative dynamics of the classical theory of fields, Acta Phys. Pol. B 7, 305 (1976), https://www .actaphys.uj.edu.pl/R/7/5/305.

[8] G. Arreaga, R. Capovilla, and J. Guven, Noether currents for bosonic branes, Ann. Phys. (Amsterdam) 279, 126 (2000).

[9] J. Guven, Covariant perturbations of domain walls in curved spacetime, Phys. Rev. D 48, 4604 (1993).

[10] J. Garriga and A. Vilenkin, Perturbations on domain walls and strings: A covariant theory, Phys. Rev. D 44, 1007 (1991).

[11] R. A. Battye and B. Carter, Second order lagrangian and symplectic current for gravitationally perturbed DiracNambu-Goto strings and branes, Classical Quantum Gravity 17, 3325 (2000).

[12] M. Mars, First and second order perturbations of hypersurfaces, Classical Quantum Gravity 22, 3325 (2005).

[13] N. Bagatella-Flores, C. Campuzano, M. Cruz, and E. Rojas, Covariant approach of perturbations in Lovelock type brane gravity, Classical Quantum Gravity 33, 245012 (2016).

[14] A. T. Fomenko and A. A. Tuzhilin, Elements of the geometry and topology of minimal surfaces in three-dimensional space, Translations of Mathematical Monographs (AMS, Providence, Rhode Island, 2005), Vol. 93.

[15] B. Murorunkwere, J. Ntahompagaze, and E. Jurua, $1+3$ covariant perturbations in power-law $\mathrm{f}(\mathrm{R})$ gravity, Eur. Phys. J. C 81, 353 (2021).

[16] S. Sahluu, J. Ntahompagaze, A. Abebe, I. de la CruzDombriz, and D.F. Mota, Scalar perturbations in $f(T)$ gravity using the $1+3$ covariant approach, Eur. Phys. J. C 80, 422 (2020).

[17] V. Kiosses and A. Nicolaidis, Second order perturbations of relativistic membranes in curved spacetime, Phys. Rev. D 89, 124016 (2014).

[18] A. L. Larsen and A. Nicolaidis, Second order perturbations of a macroscopic string: Covariant approach, Phys. Rev. D 63, 125006 (2001).

[19] S. Ryu and T. Takayanagi, Aspects of holographic entanglement entropy, J. High Energy Phys. 08 (2006) 045.

[20] V.E. Hubeny, M. Rangamani, and T. Takayanagi, A covariant holographic entanglement entropy proposal, J. High Energy Phys. 07 (2007) 062.

[21] N. Bao, C. Cao, S. Fischetti, and C. Keeler, Towards bulk metric reconstruction from extremal area variations, Classical Quantum Gravity 36, 185002 (2019).

[22] N. Bao, C. Cao, S. Fischetti, J. Pollack, and Y. Zhong, More of the bulk from extremal area variations, Classical Quantum Gravity 38, 047001 (2021).

[23] E. Engelhardt and E. Fischetti, Surface theory: The classical, the quantum, and the holographic, Classical Quantum Gravity 36, 205002 (2019).
[24] R. Martens and K. Koyama, Brane-world gravity, Living Rev. Relativity 13, 5 (2010).

[25] T. Regge and C. Teitelboim, General relativity à la string: A progress report, in Proceedings of the Marcel Grossman Meeting, Trieste, Italy, 1975, edited by R. Ruffini (NorthHolland, Amsterdam, 1977), 77.

[26] M. Deserno, Fluid lipid membranes: From differential geometry to curvature stresses, Chem. Phys. Lipids $\mathbf{1 8 5}$, 11 (2015).

[27] R. Capovilla, A simultaneous variational principle for elementary excitations of fluid lipid membranes, J. Phys. Commun. 2, 025003 (2018).

[28] R. Capovilla, Elastic bending energy: A variational approach, J. Geom. Symmetry Phys. 45, 1 (2017).

[29] J. Armas, J. Hartong, E. Have, B. F. Nielsen, and N. A. Obers, Newton-Cartan submanifolds and fluid membranes, Phys. Rev. E 101, 062803 (2020).

[30] J. Armas and J. Tarrío, On actions for (entangling) surfaces and DCFTs, J. High Energy Phys. 04 (2018) 100.

[31] R. Wald, General Relativity (University Chicago Press, Chicago, 1984).

[32] I. M. Gelfand and S. V. Fomin, Calculus of Variations (Prentice-Hall, Englewood Cliffs, NJ, 1963).

[33] M. Kot, A First Course in the Calculus of Variations (AMS, Student Mathematical Library, Providence, Rhode Island, 2014), Vol. 72.

[34] S. L. Bażański, Dynamics of relative motion of test particles in general relativity, Ann. Inst. H. Poincaré A 27, 145 (1977), http://www.numdam.org/item/AIHPA_1977_27_2_ $145 \_0 /$.

[35] M. Cruz and E. Rojas, Born-Infeld extension of Lovelock brane gravity, Classical Quantum Gravity 30, 115012 (2013).

[36] S. L. Bażański, Hamilton-Jacobi formalism for geodesics and geodesic deviations, J. Math. Phys. (N.Y.) 30, 1018 (1989).

[37] R. Capovilla and G. Cruz, A covariant simultaneous action for branes, Ann. Phys. (Amsterdam) 411, 167959 (2019).

[38] M. Giaquinta and S. Hildebrandt, Calculus of Variations (Springer, New York, 2004).

[39] E. Y. López, Perturbaciones de segundo orden de Branas, M.Sc. thesis, CINVESTAV-IPN, 2018.

[40] P. A. M. Dirac, An extensible model of the electron, Proc. R. Soc. A. 268, 57 (1962).

[41] Y. Nambu, Duality and Hadrodynamics; Lectures at the 1970 Copenhagen High Energy Symp, in Broken Symmetry: Selected Papers of Y. Nambu, edited by T. Eguchi and K. Nishijima (World Scientific, Singapore, 1995), pp. 280-301.

[42] T. Gotō, Relativistic quantum mechanics of one-dimensional mechanical continuum and subsidiary condition of dual resonance model, Prog. Theor. Phys. 46, 1560 (1971). 